\section{Merck's Rosetta stone}

\section{By Lev Osherovich, Senior Writer}

Four papers published in Nature Genetics, Nature and Public Library of Sciences Biology provide a compelling proof of concept for the use of integrative genomic analysis to drive the discovery of genes underlying complex diseases such as obesity and other metabolic disorders. Genomics companies told SciBX that the approach described in these papers had been considered technically unfeasible, but now its application could significantly accelerate marker and target discovery.

Indeed, the integrative approach reported in the papers, which was developed by Merck \& Co. Inc. subsidiary Rosetta Inpharmatics LLC, is already shaping the pharma company's drug discovery efforts.

Genome mining for new therapeutic targets often focuses either on genotype mapping or gene expression analysis. Due to the volume and complexity of information in genome-wide data sets, researchers have had only limited success at integrating these different levels of genomic information.

By contrast, the new studies paint the clearest picture yet of how the many facets of genomic structure and regulation fit together to influence disease. ${ }^{1}$

Three of the studies were from combined industry and academic teams led by Eric Schadt, executive scientific director of genetics at Rosetta, whereas the fourth study was co-led by Kari Stefansson, CEO of deCODE genetics Inc.

All four studies integrate genotype and gene expression analysis with additional data, including transcription factor binding sites and protein-protein interactions as well as physiological measurements. Three studies used data from yeast, mice and human tissue samples. The study coauthored with Stefansson analyzed human clinical data.

\section{Master genes}

The main method used in the four studies was expression quantitative trait locus (eQTL) mapping, which identifies chromosomal regions that regulate the expression of genes associated with disease. ${ }^{2}$

Conventional QTL mapping identifies SNPs that correlate with elevated disease risk, pointing to hereditary factors that influence disease, but it does not shed light on how these factors interact with each other in disease.

In contrast, $\mathrm{eQTL}$ analysis aims to map markers near master regulator genes that control the expression of large groups of other genes involved in disease. eQTL mapping can identify gene networks and their master switches even in healthy subjects, making it particularly useful for studying complex and environmentally influenced diseases like metabolic syndrome.

eQTL mapping gathers gene expression data using mRNA microarrays and then looks for correlations between individual SNPs and genome-wide patterns of gene expression indicative of disease.

According to Schadt, eQTL overcomes the limits of conventional gene mapping by uncovering pivotal genes in diseases that are not purely heritable, but are influenced by multiple genes. Although QTL studies can identify risk loci, eQTL can unravel the relationships between them.

"Complex traits such as common human diseases or drug response result from many genes interacting in complex ways," Schadt told SciBX. "You have to look beyond DNA variation into a space that incorporates both DNA variation and gene activity."

Like conventional QTL mapping, eQTL points to fairly broad chromosomal intervals but doesn't directly identify candidate genes. To find the lynchpin genes, Schadt's team gathered additional experimental, clinical and genomic data and then combined the results to generate theoretical models of genetic networks that best explained the data.

Schadt told SciBX that Merck's acquisition of Rosetta in 2001 allowed the company to build and staff a customized computing facility to handle the massive amount of data involved in the studies.

"Getting and managing the data is technically challenging," he said. "The number of combinations you have to consider is large. We're getting into terabytes of data" per single experiment, he noted.

\section{Yeast and mouse proof}

As a proof of principle, Schadt's team deployed the eQTL technique in yeast and in mice. In these organisms, the genetic variation needed to create an eQTL map can be generated by crossing unrelated strains and analyzing their offspring. The yeast study appeared in Nature Genetics, ${ }^{3}$ whereas the mouse study was published in Nature. ${ }^{4}$

In the yeast study, Schadt's team used pre-existing DNA sequence and gene expression data from a panel of genetically related yeast strains to identify 'hot spots' - areas of the genome with particularly strong eQTL signals.

To find the master genes hidden in the hot spots, the team computationally compared the eQTL data with published information about protein-protein interactions and transcription factor binding sites. This analysis yielded a list of likely master regulator genes involved in yeast metabolism.

Indeed, strains lacking these critical regulators showed changes in the expression of the target genes predicted by the analysis.

Schadt's team also used eQTL in mice to identify genes that influence obesity and other metabolic traits. Focusing on a portion of chromosome 1 that previously had been implicated in metabolic disease, the researchers conducted an eQTL scan of adipose tissue using both gene expression data and measurements of weight, fat mass and cholesterol levels.

The most likely suspects turned out to be a network of genes expressed in macrophages, a type of innate immune cell. Previous studies had sug- 
gested that macrophage infiltration of adipose tissue was linked with obesity, but the mechanisms of this process were unknown. ${ }^{5}$

Schadt's team termed the group of these coordinately regulated genes the macrophage-expressed metabolic network (MEMN).

To test their predictions, the team disrupted several of the MEMN genes and measured the effect on obesity-associated traits. Mice lacking one copy of lipoprotein lipase ( $L p l$ ) —one of the MEMN genes-had 22\% higher fat-mass-to-lean-mass ratio than wild-type littermates. Likewise, overexpression of another protein in the network, $\beta$-lactamase, made mice $20 \%$ fatter than wild-type controls.

The strongest master regulator eQTL signal in the mouse study came from another MEMN gene, which encodes a newly discovered protein phosphatase called protein phosphatase 1 (formerly 2C)-like (Ppm1L; Ppm1-like). Schadt's team found that Ppm1L knockouts displayed many characteristics of metabolic syndrome, including faster weight gain, higher adult weight and higher fat mass than wild-type controls. Ppm1L knockouts had hyperinsulinemia, high glucose tolerance and higher blood pressure than wild-type controls, all of which are characteristics of human metabolic syndrome.

Ppm1L thus could be a target for drug development to treat metabolic syndrome. Alternatively, Ppm1L knockout could be a new mouse model for the disorder.

\section{From mice to men}

Concurrent with the mouse study, Schadt's team collaborated with deCODE to tease out human metabolic syndrome genes. The work, reported in the second Nature article, involved eQTL analysis of tissue samples from hundreds of Icelanders. ${ }^{6}$

deCODE uses Iceland's complete genetic and medical information to hunt for disease-associated genes and to develop diagnostics.

The Merck and deCODE team analyzed the expression of $84 \%$ of the human genome in adipose tissue and blood from 1,675 Icelanders, integrating these data with genotype analysis of 1,732 microsatellite markers, which are repetitive noncoding DNA sequences often used in gene-mapping studies.

An additional level of precision came from an analysis of 317,503 SNPs in a subset of the subjects. As the hereditary relationships of all Icelanders are known, eQTL signals were also correlated to patterns of inheritance, thus improving the method's accuracy.

The team also analyzed clinical data such as body mass index (BMI), percentage body fat and waist-to-hip ratio from each individual. These metrics have previously been used to describe obesity and to predict susceptibility to metabolic syndrome. ${ }^{7}$

By computationally merging the clinical, genotypic and eQTL data, the team homed in on the genes most likely to underlie obesity. In fact, many candidate genes belonged to the MEMN, just as in mice. Variation in expression of most MEMN genes correlated with BMI variation, although the correlation with percent body fat was not as strong.

The Nature study did not reveal whether human homologs of the three mouse genes described in Schadt's Nature paper were also relevant in human obesity. However, according to deCODE's website, one of three genes identified in the now completed collaboration is the target of a preclinical compound being developed by Merck. The pharma company would not disclose the target or development status of the compound.

deCODE and Merck finished joint work exploring the human genome for obesity targets in 2005 .

Finally, a study of eQTLs in human livers by Schadt and collaborators at five academic institutions, published in PLoS Biology, identified Sortilin 1 (SORT1) and Cadherin EGF LAG seven-pass G-type receptor 2 (CELSR2) as candidate susceptibility genes influencing coronary artery disease and plasma low-density lipoprotein (LDL) cholesterol levels. ${ }^{8}$

Schadt said the method could lead to faster transition from gene discovery to therapeutic development. He noted that the Rosetta team's gene discovery methods work hand in hand with the human gene-targeting technologies being developed by another Merck subsidiary, siRNA Therapeutics Inc.

"Once you have a network that defines a disease state, you can go into a human experimental study and hit that network with different [short interfering] RNAs," he said. "Merck's siRNA technology lets us start thinking about multiple nodes at once and going into experimental clinical studies."

\section{Other perspectives}

Genomics companies told SciBX that Schadt's studies are proof of principle of methods previously thought to be technically unfeasible, and that they will significantly accelerate marker and target discovery.

"Genome-wide association studies with whole-genome SNP platforms and gene expression are not new," said Tod Klingler, VP of information sciences at molecular diagnostics company XDx Inc. "The innovation is the series of informatics and data analysis."

He added: "Network modeling is really brand new. These are significant experiments."

In genomics studies of this size, said Klingler, "you often have to worry about false discovery rates." However, he said, Schadt's method of grouping genes in networks and pathways greatly improves the chances of "finding the signal in the noise."

XDx markets AlloMap, a molecular expression screening service for diagnosing risk of acute cellular rejection after cardiac transplant.

"Merck's approach could be used to better organize the gene expression results that we're getting," said Russ Dietrich, XDx's director of molecular immunology.

Gualberto Ruaño, president and CEO of Genomas Inc., told SciBX that Schadt's integration of clinical data into the eQTL analysis makes the work highly innovative.

"Technically, it's a tour de force to do so many biopsies of so many individuals," he said. "Looking at gene expression is a step in the right direction."

Genomas uses genomic marker analysis together with clinical measurements to help doctors manage risks of side effects in cardiac and neuropsychiatric therapy, said Ruaño.

Both Klingler and Ruaño agreed the most immediate application of Schadt's methods is to discover diagnostic markers and to understand the organization of gene networks influenced by disease.

However, Ruaño cautioned that the genes identified as relevant to a disease process will likely be relevant to other processes involved in 


\section{COVER STORY}

normal physiology as well. "These genes are too far upstream” to typically be useful targets, he said.

According to Merck spokesperson Caroline Lappetito, the company "has filed patent applications on some of the broader concepts developed by Schadt but has chosen not to protect most of the more detailed methods and algorithms being developed by his group."

"Merck will seek to protect novel targets and biomarkers identified as a result of using the unique methods developed by Schadt's group," she added.

\section{REFERENCES}

1. McCarthy, M.I. et al. Nat. Rev. Genet. 9, 356-369 (2008)

2. Gilad, Y. et al. Trends Genet.; published online July 1, 2008; doi:10.1016/ j.tig.2008.06.001

3. Zhu, J. et al. Nat. Genet.; published online June 15, 2008; doi:10.1038/ng.167

Contact: Eric Schadt, Rosetta Inpharmatics LLC, Seattle, Wash. e-mail: eric_schadt@merck.com
4. Chen, Y. et al. Nature; published online March 16, 2008; doi:10.1038/nature06757

Contact: Eric Schadt, Rosetta Inpharmatics LLC, Seattle, Wash. e-mail: eric_schadt@merck.com

5. Heilbronn, L.K. \& Campbell, L.V. Curr. Pharm. Des. 14, 1225-1230 (2008)

6. Emilsson, V. et al. Nature; published online March 16, 2008; doi:10.1038/ nature06758

Contact: Kari Stefansson, deCODE genetics Inc., Reykjavik, Iceland e-mail: kari.stefansson@decode.is

7. Haffner, S.M. Obesity (Silver Spring) 14, 121S-127S (2006)

8. Schadt, E.E. et al. PLoS Biol.; published online May 6, 2008; doi:10.1371/journal.pbio.0060107

Contact: Eric Schadt, Rosetta Inpharmatics LLC, Seattle, Wash. e-mail: eric_schadt@merck.com

COMPANIES \& INSTITUTIONS MENTIONED

deCODE genetics Inc. (NASDAQ:DCGN), Reykjavik, Iceland Genomas Inc., Hartford, Conn.

Merck \& Co. Inc. (NYSE:MRK), Whitehouse Station, N.J.

Rosetta Inpharmatics LLC, Kirkland, Wash.

siRNA Therapeutics Inc., San Francisco, Calif.

XDx Inc., Brisbane, Calif. 